\title{
Design of Flight and Engine Warnings and Parameter Display System
}

\author{
Mahasweta Bhattacharya ${ }^{1}$ \\ ${ }^{1}$ (Electronics and Communication Engineering, West Bengal University of Technology, West Bengal, India,
}

\begin{abstract}
Aircraft Cockpit Instrument System normally has conventional electromechanical instruments for Flight and Engine parameters of prime importance to the crew. These instruments include flight instruments like, Vertical Speed Indication (VSI), Standby Altitude Indicator, Combined Speed Indicator, Altimeter, Radio Magnetic Indicator (RMI). The Engine and Fuel instrument include RPM indicator, Turbine Exhaust Gas Temperature (TGT) indicator, Nozzle Position indicator, Fuel Flow meter, Fuel content indicator. In addition to flight and engine parameter indicators, a gyro magnetic compass (GMC) and Hydraulic pressure indicator are also required.

In modern time aircraft, upgraded avionics replaces this entire instrument by integrated flight and engine parametric display and warning system. The flight parameters are received from Air Data, Altitude and Heading Reference System (ADAHRS). The ADAHRS shall have a serial interface (RS 422 or ARINC 429) from FEW-DPS. The Engine parameters and hydraulic pressure are directly acquired by Data Acquisition block of FEW-PDS. After the application of the computational algorithm in FEW-PDS, the corresponding value of parameters are displayed in LCD based soft instruments display. FEW-PDS shall also generate the discrete signal for different warnings based on algorithms as part of application $s / w$ running on the main microcontroller of FEW-PDS. The scope of project is a Hardware Design for Lab Model of FEW-PDS.
\end{abstract}

Keywords: ARINC429, TGT, Nozzle Position, Engine RPM, Fuel Flow Rate, Hydraulic Pressure, Serial Peripheral Interface

\section{Introduction}

This project aims at the hardware design of the lab model of the FEW-PDS. A FEW-PDS mainly displays the several flight and engine parameters and also warnings when the parameters go beyond their permissible limits. This parameters are received from the Air Data Altitude and Heading Reference System also known as the ADAHRS. The ADAHRS is serially interfaced with the FEW-PDS by means of ARINC 429 bus. The FEW-PDS is based on the 8051-coreprocessor MSC1212 from Texas Instruments. The altitude and heading references are directly sent by the ARINC data bus to the FEW-PDS. Apart from the altitude and the heading parameters, there are also several other parameters like the Engine Parameters and the Hydraulic Pressure. These parameters are directly acquired by the Data Acquisition Block of FEW-PDS implemented through the MSC1212. Of the several parameters, 5 parameters have been considered here. They are as follows:

\author{
Turbine Gas Temperature \\ Engine RPM \\ Nozzle Position \\ Fuel Flow Rate \\ Hydraulic Pressure
}

The parameters obtained by the Data Acquisition block are displayed on the LCD and the FEW-PDS also generates discrete signal corresponding to the parameter values. The FEW-PDS includes an integrated system of the Data Acquisition block and a Display Unit where the Data Acquisition Block is interfaced with a Virtex 6 FPGA kit where an LCD display shows the parameter values as well as the warnings when the values are beyond the permissible range. The Figure 1.1 in the next page shows the FEW-PDS system that has been designed. 


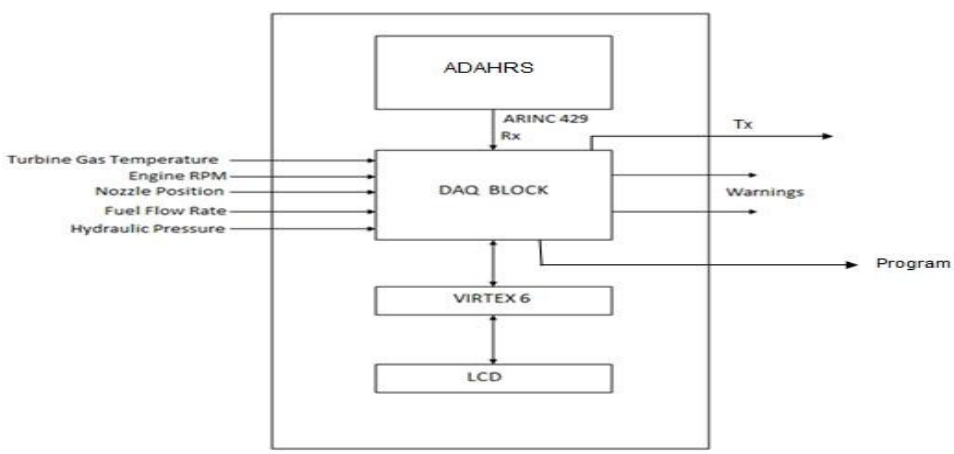

Figure 1: Block Diagram of FEW-PDS System

\section{Data Acquisition Block}

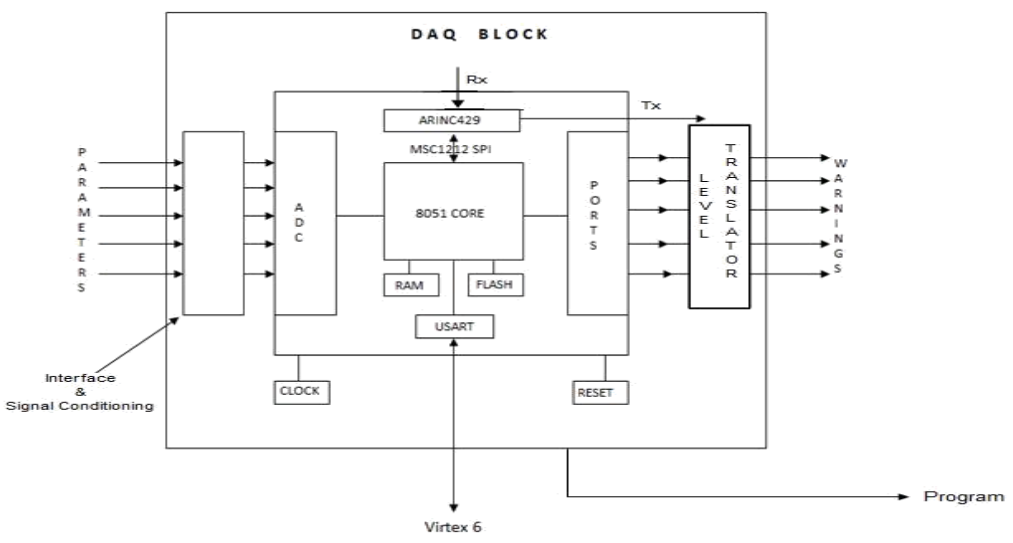

Figure 2: Block Diagram of Data Acquisition

The data acquisition block shown in Figure 2.1 uses MSC1212 microcontroller. The microcontroller has inbuilt ADC, USART, SPI and DIO. It also consists of inbuilt FLASH for Program and RAM for program execution and data handling. One of main purpose of selecting particular microcontroller is that it includes that it include the required high precession 16 bit ADC and required SPI and USART interface. It is built around enhanced 8051 core. The SPI interface is used to connect the ARINC 429 controller. Air data, Attitude and heading reference system (ADAHRS) is connected to FEW-PDS on ARINC429 Data bus. Besides that Data Acquisition block acquires in the several engine parameters and transmit on serial port for display. It also generates gives the respective discrete warning when it goes beyond the acceptable limit. The microcontroller with this feature that has been used in our application is the MSC1212Y5.

\section{Ii.1 Jj. Serial Peripheral Interface Between Arinc 429 And} Msc1212

The MSC1212Y5 has the provision for Serial Peripheral interface or SPI. SPI allows the microcontroller to exchange data between itself and the periphery. The SPI is a full-duplex, synchronous, character-oriented channel that supports a four-wire interface (receive, transmit, clock, and slave select). The SPI block consists of transmitter and receiver sections, an independent baud rate generator, and a control unit. The transmitter and receiver sections use the same clock. During an SPI transfer, simultaneous transfer of eight bits of data occurs from a master to a slave, and another eight bits of data from the slave to the master. The MSC1212 can be programmed to behave as a master or a slave and uses four signals to coordinate transfers. The four signals are:

1. SS - Slave Select (shared with P1.4/INT2)

2. MOSI- Master Out/Slave In (shared with P1.5/INT3)

3. MISO- Master In/Slave Out (shared with P1.6/INT4/SDA)

4. SCK- SPI Clock(shared with P1.7/INT5 /SCL)

In our project, we have used the SPI interfacing to interface the ARINC 429 with the MSC1212 for data exchange between them. The ARINC 429 receives the Air Data, Attitude and Heading Reference data from 
ADAHRS which is interfaced to the MSC1212 microcontroller of the Data Acquisition Block through SPI interface.

The ARINC 429 data bus is interfaced with the microcontroller by means of a special chip from Holt Integrated Circuits Inc. named as the HI3593. HI3593 is specifically used for interfacing a SPI enabled microcontroller to the ARINC429 data bus. The HI3593 is a ARINC429 controller, providing ARINC429 transmit and receive channels.

The ARINC429 acts as the slave to the master MSC1212 while operating in the SPI mode. For operation in the SPI mode, The 4 wire SPI lines of MSC should be properly connected with the SPI lines of HI3593 for serial communication.

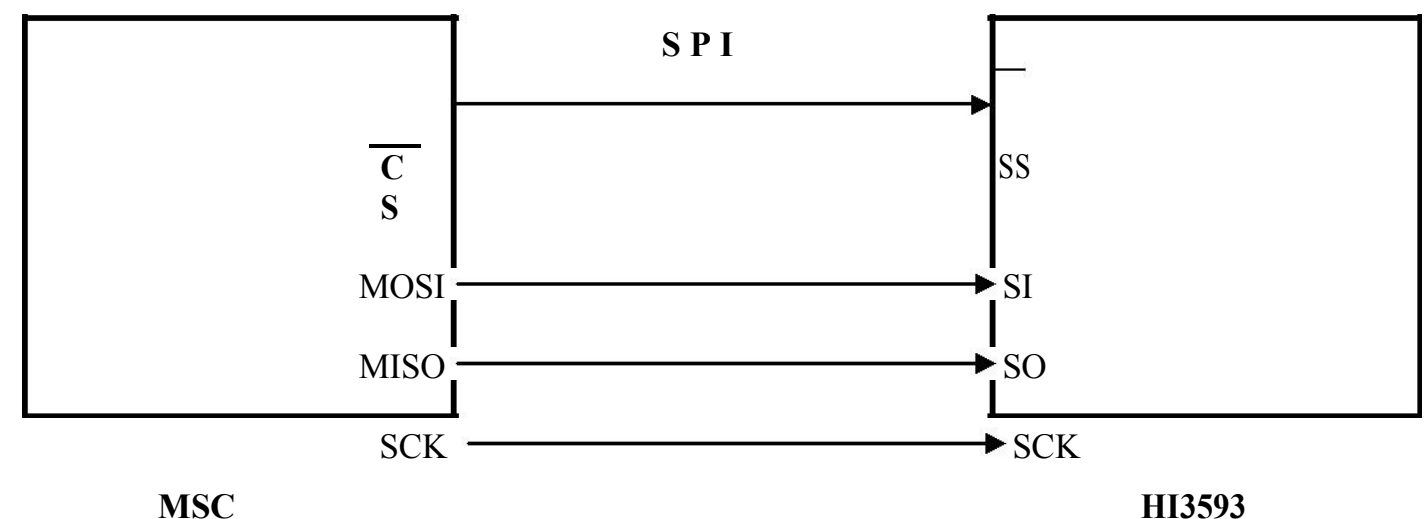

Figure 3: Block Diagram of ARINC429 and Microcontroller SPI Interface The connection should be made as follows:

1. SCK of the HI3593 should be connected to the SCK of MSC so that both the ARINC databus and the controller of the data acquisition block are in synchronization. Otherwise the timing of data transmission between both the systems will fail to match and thus would lead to erroneous data transmission.

2. The SS line of the ARINC chip is known as the Slave select. When the master i.e MSC has multiple slaves then it needs to select the particular slave with which it wants to communicate. Our application requires the MSC to communicate with the ARINC, thus we connect the Chip Select line of the controller to the Slave Select Line of the ARINC chip in order to ensure serial communication between them.

3. SI line symbolizes Slave Input which denotes the line through which the slave that is the ARINC bus will receive data from the master i.e MSC. So the SI line is connected to the MOSI line of the MSC which ensures that the output data from the master goes as the input data into the slave.

4. SO line symbolizes Slave Output which denotes the line through which the slave i.e the ARINC bus will send data to the master i.e MSC. So the SO line is connected to the MISO line of the MSC which ensures that the input data to the master comes as the output data from the slave.

Thus the 4 lines of the ARINC chip are connected to the 4 corresponding lines of the controller thereby establishing serial communication between them. Separate RESET and Oscillator chips have been used.

\section{PARAMETER ACQUISITION FROM SENSORS}

\section{a) Analog to Digital Converter}

$\mathrm{ADC}$ has its own high precision analog to digital subsystem. The analog to digital subsystem block of MSC1212 has been used primarily in the project. It is used to acquire the direct parameters like TGT, Nozzle position, Engine RPM and Fuel Flow rate. An input interface and required signal conditioning is implemented for each signal to convert the input signal to appropriate voltage level for feeding to inputs to ADC of microcontroller. 


\section{b) Parameter Acquisition}

In this lab model of FEW-PDS 5 direct parameters of importance are considered for implementation.

The 5 parameters are:-

1. Turbine Gas Temperature(TGT)

2. Engine RPM (RPM)

3. Nozzle Position (NZ)

4. Fuel Flow Rate ( FFR)

5. Hydraulic Pressure (HY)

Parameter definition (Signal type and range) are given below in the table:

\begin{tabular}{|c|c|c|c|}
\hline \multirow[t]{2}{*}{ PARAMETERS } & \multicolumn{2}{|l|}{ RANGE } & \multirow[t]{2}{*}{ TYPE } \\
\hline & MIN & MAX & \\
\hline $\begin{array}{l}\text { Turbine Gas } \\
\text { Temperature(TGT) }\end{array}$ & $0^{0} \mathrm{C}$ & $750^{\circ} \mathrm{C}$ & $\begin{array}{l}\text { Temperature Measurement, Type } \mathrm{J} \text { thermo } \\
\text { couple }\end{array}$ \\
\hline $\begin{array}{l}\text { Engine RPM } \\
\text { (RPM) }\end{array}$ & $\mathrm{OHz}$ & $77 \mathrm{~Hz}$ & Frequency \\
\hline $\begin{array}{l}\text { Nozzle Position } \\
\text { (NZ) }\end{array}$ & $1.4 / 28$ & $26.6 / 28$ & $\begin{array}{l}\text { Ratio metric, voltage value } 1.4 \text { volt to } 26.6 \\
\text { volt with excitation of } 28 \mathrm{~V} \text {. }\end{array}$ \\
\hline $\begin{array}{l}\text { Fuel Flow } \\
\text { Rate(FFR) }\end{array}$ & $0 \mu \mathrm{A}$ & $1025 \mu \mathrm{A}$ & Current to Voltage \\
\hline $\begin{array}{l}\text { Hydraulic Pressure } \\
\text { (HY) }\end{array}$ & $2.66 / 28$ & $25.34 / 28$ & $\begin{array}{l}\text { Ratio metric, voltage value } 2.66 \text { volt to } \\
25.34 \text { volt with excitation of } 28 \mathrm{~V} \text {. }\end{array}$ \\
\hline
\end{tabular}

Table 1 Engine Parameters And Hydraulic Pressure Range

\section{i. Turbine Gas Temperature}

Turbine Gas Temperature is one of the important engine parameters. This indicates the Turbine Exhaust Gas Temperature. The FEW-PDS design specific to this application takes a Temperature limit from 00C to 7500C (J type thermocouple). At front end, a interface IC AD594 is used for taking the J type thermocouple input and provide 0 to 7.5 volt voltage output. Taking the exceeding limit in the design the subsequent ckt is design for 0 to $10 \mathrm{~V}$ range. The voltage is divided by 4 and given to ADC for conversion in the range of $0-2.5 \mathrm{~V}$.

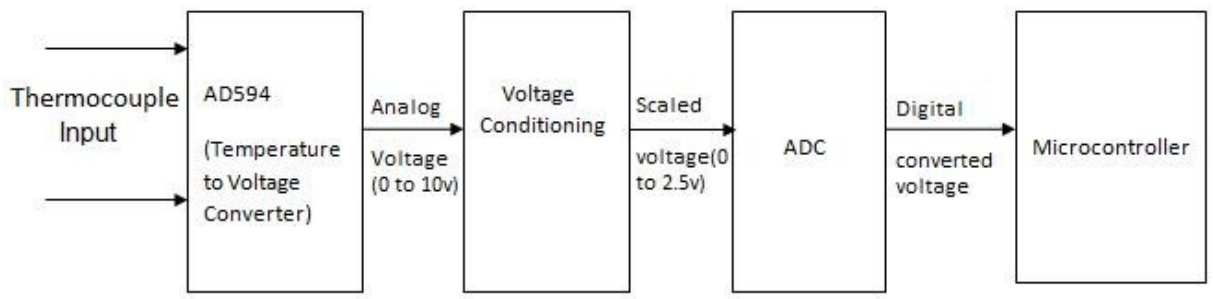

Figure 4: Block Diagram of Turbine Gas Temperature Acquisition 
The principle of thermocouple measurement mainly state that the difference in thermal characteristics between the two metals of the thermocouple creates a difference in electron flow and hence causes a voltage difference. For this application we have used an IC chip for thermocouple interface and providing analog voltage in the range measurable by ADC. This IC chip used for this purpose is AD594. The AD594 uses a +5V supply for measuring a temperature range of 0-3000C. But TGT in fighter aircraft can reach at a temperature of about $7500 \mathrm{C}$ which is considered as critical. Therefore, for this application the temperatures beyond $7500 \mathrm{C}$ is also required to be measure. The working temperature range can be increased beyond $7500 \mathrm{C}$ by changing the supply voltage from $5 \mathrm{~V}$ to $15 \mathrm{~V}$. Thus for our design $+15 \mathrm{~V}$ supply is used to power our AD594.

\section{ii. Engine RPM}

Engine RPM is one of the important engine parameters. This is indicated in revolution per minute for the engine. The FEW-PDS design specific to this application takes a frequency signal for $0 \mathrm{~Hz}$ to $77 \mathrm{~Hz}$. Here also we need to convert the frequency into its corresponding analog voltage which is fed to the analog input line 2 i.e AIN1 for its conversion to corresponding digital value. Block diagram of frequency to voltage converter is shown in the diagram below. For our application the frequency to voltage conversion is done with the help of a IC chip LM2917.

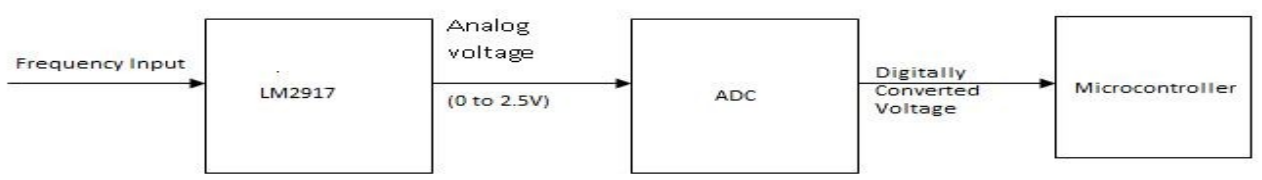

Figure 5: Block Diagram of Engine RPM Acquisition

The main block diagram of the frequency to voltage converter is shown in figure.

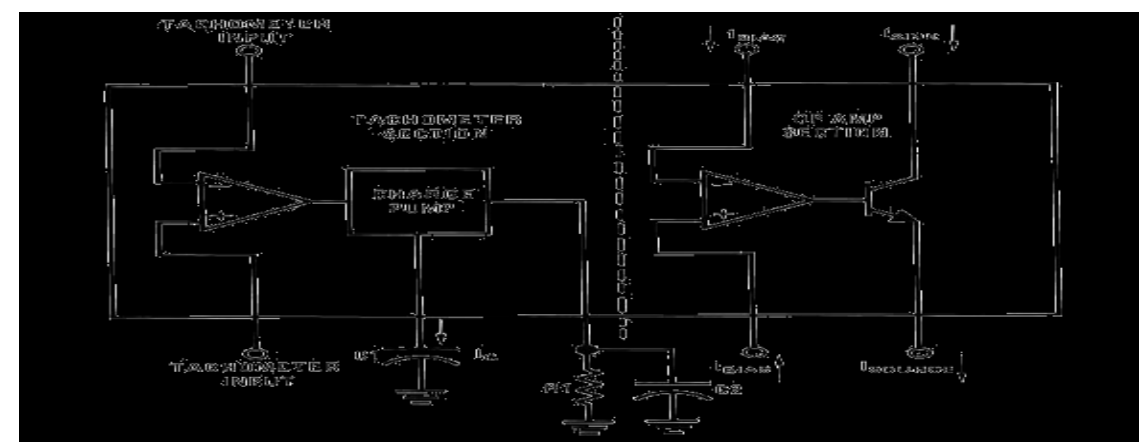

Figure 6: Internal Block Diagram of LM2917

It consists of a charge pump where the input frequency is converted to a dc voltage. To do this we require one timing capacitor, one output resistor, and an integrating or filter capacitor. When the input stage changes state (due to a suitable zero crossing or differential voltage on the input) the timing capacitor is either charged or discharged linearly between two voltages whose difference is $\mathrm{VCC} / 2$. Then in one half cycle of the input frequency or a time equal to $1 / 2 \mathrm{fIN}$, the change in charge on the timing capacitor is equal to $\mathrm{VCC} / 2 \times \mathrm{C} 1$. $\mathrm{V} 0=\mathrm{VCC} \times \mathrm{fIN} \times \mathrm{C} 1 \times \mathrm{R} 1 \times \mathrm{K}$, where $\mathrm{K}$ is the gain constant and typically of the value of 1 . $\mathrm{C} 2$ depends on the amount of the ripple voltage allowable and the required response time. R1 should be chosen with utmost care as a very low R1 would cause an error current whereas a larger value become a significant portion of the output impedance which degrades the linearity. The conventional value of R1 is given by the equation:

For the measurement of engine RPIM we have chosen the maximum limit of the RPM to be $77 \mathrm{~Hz}$ and the corresponding voltage to $f(2,5 \mathrm{~V}$ which is fed as the analog voltage input to the ADC of the microcontroller. The instrument can respond perfectly tifl $28 \mathrm{~V}$. Fut we know that frequency response causes amplitude of signal to diminish at the corner frequency and thus we need to take a higher frequency value for our design which will help to avoid the effect of corner frequency so that power reduction does not occur at the corner frequencies we have chosen the frequency to be $100 \mathrm{~Hz}$ for calculating the C1. The LM2917 draws a typical current of $180 \mu \mathrm{A}$ at the output. So we have,

$\mathrm{f}_{\mathrm{MAX}}=100 \mathrm{~Hz}, \mathrm{Vcc}=12 \mathrm{~V}, \mathrm{I}_{2}=180 \mu \mathrm{A}$

Therefore, 


$$
C_{1}=\frac{I_{2}}{f_{M A X} \times V_{C C}}
$$

Substituting the given values we get, $\mathrm{C}_{1}=0.15 \mu \mathrm{A}$.

For calculating R1, $\mathrm{V}_{0}=2.5 \mathrm{~V}$. Our maximum input frequency can be $77 \mathrm{~Hz}$. So $\mathrm{f}_{\mathrm{IN}}=77 \mathrm{~Hz}$. $\mathrm{C} 1=0.15 \mu \mathrm{A}$.

Therefore,

$$
R_{1}=\frac{V_{0}}{f_{I N} \times V_{C C} \times C_{1}}
$$

which gives $\mathrm{R} 1=18.04 \mathrm{~K} \Omega$. $\mathrm{C} 2$ has been chosen as $1 \mu \mathrm{F}$. These are the values of $\mathrm{R} 1$ and $\mathrm{C} 1$ that we have chosen for our application.

\section{iii. Nozzle Position}

Nozzle Position is a ratiometric analog parameter. $28 \mathrm{~V}$ DC is given as a excitation voltage to the pot. The pot mainly consists of a resistance $(500 \mathrm{ohm})$ with a wiper whose movement is based on the nozzle position. Movement of the wiper varies the voltage and thus the nozzle position is measured.

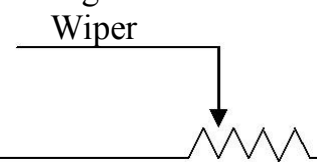

Figure 7: Schematic Diagram of Wiper Indicating Nozzle Position

The wiper wipes over the resistance in accordance with the nozzle position which in turn changes the voltage. This voltage corresponds to the nozzle position and it is acquired by ADC after proper signal conditioning. In our application the ADC is a part of the microcontroller which converts the voltage to its corresponding digital value.

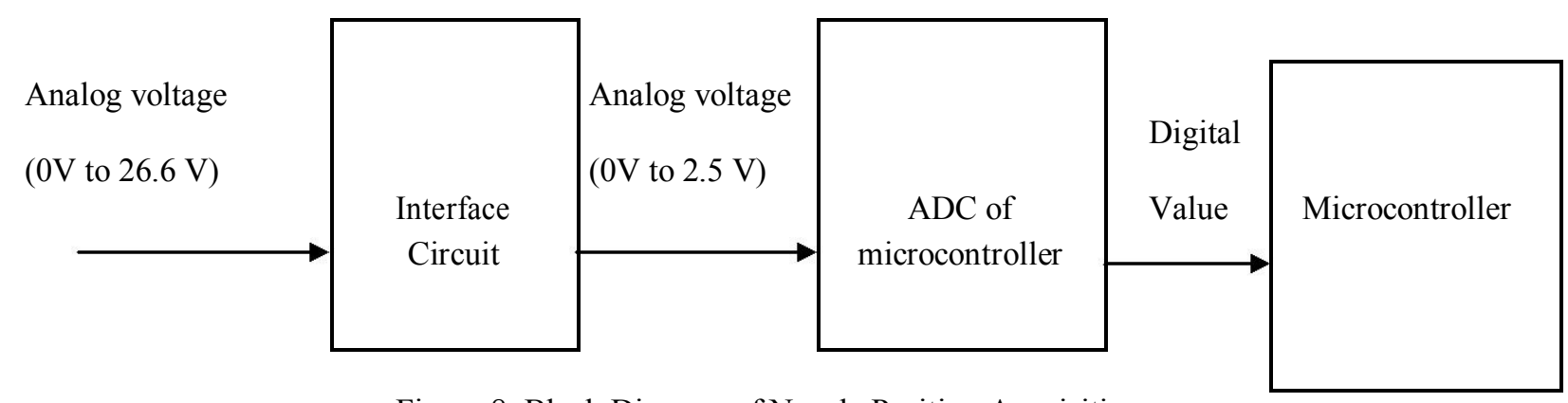

Figure 8: Block Diagram of Nozzle Position Acquisition

\section{Interface Circuit}

The excitation voltage to the pot is $28 \mathrm{~V}$ DC. The wiper position varies between $0 \mathrm{~V}$ to $26.6 \mathrm{~V}$ which is to be supplied to the ADC. But the ADC of the microcontroller has the maximum operating voltage limit of + $2.5 \mathrm{~V}$. So it cannot be directly applied to the microcontroller. Hence, the wiper input is scaled down between + $2.5 \mathrm{~V}$ range and then applied to the $\mathrm{ADC}$ of the microcontroller. This is achieved with the help of a gain adjustable OPAMP based instrumentation amplifier. Same is shown below.

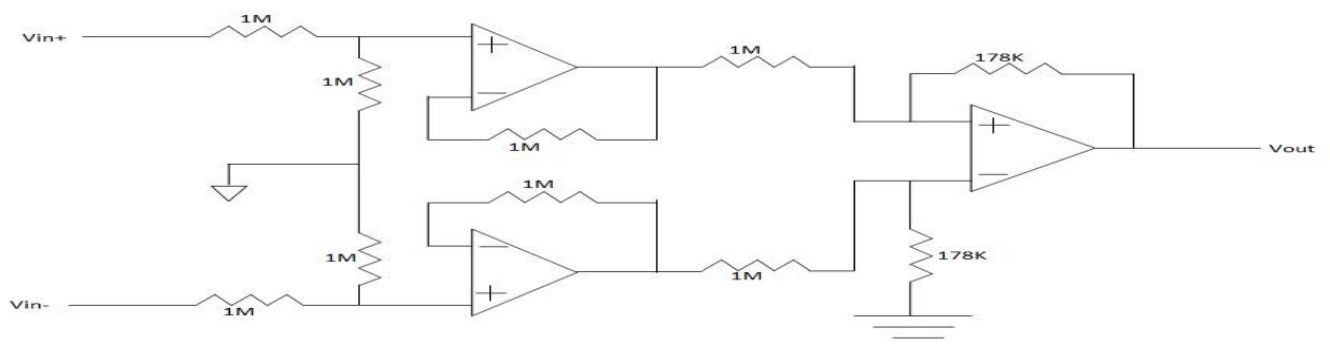

Figure 9: Circuit Diagram of Voltage Conditioning to 2.5V 
Now for our application, to divide the $28 \mathrm{~V}$ to our desired voltage level we have used the TL074 IC OP-AMP.

\section{iv. Fuel Flow Rate}

This parameter is the fuel flow rate where the fuel probe measures the flow rate. The sensor present in the probe generates a current signal. This current signal is measured to calculate the fuel flow rate. In our application the maximum current value is $1025 \mu \mathrm{A}$. This signal is first converted into voltage and then applied to the microcontroller ADC. The basic circuit diagram of a current to voltage converter is given as:

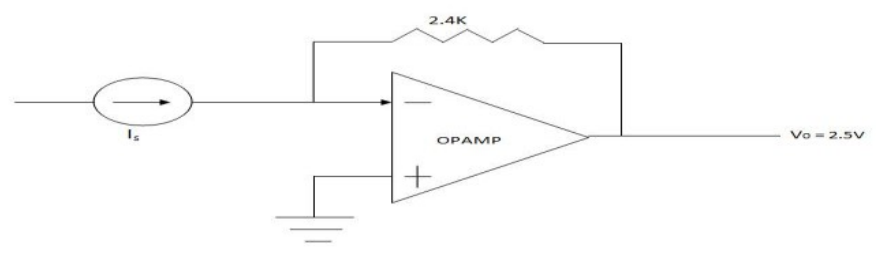

Figure 10: Circuit Diagram of Fuel Flow Rate Data Conditioning

Now, from the circuit it is evident that $V_{0}=I_{s} \times R$

For our application, Is $=1025 \mu \mathrm{A}$.

Also, since we need to supply the voltage to ADC which works at $2.5 \mathrm{~V}$ hence, $\mathrm{V} 0=2.5 \mathrm{~V}$.

Therefore,

By substituting the values we get, $\mathrm{R}=2.439 \mathrm{~K} \Omega$. Thus we chose $\mathrm{R}$ as $2.4 \mathrm{~K} \Omega$.

For this application also the TL074 is used as the OP-AMP.

Hydraulic Pressure

Hydraulic Pressure parameter is measured in bar. It is sensed by a sensor which generates an analog voltage corresponding to the Hydraulic Pressure. So by reading this voltage, hydraulic pressure can be measured. The sensor used for measuring the hydraulic pressure is same as used for the measurement of nozzle position. Same type of circuit is used to acquire this parameter as is used in the case of nozzle position.

\section{Warning Generation As Discretes}

The FEW-PDS also aims at not only the parameter readings but also generating warnings when the value of any one of the parameter goes beyond the permissible limit. In general for aircraft parameters, a warning is generated when a parameter's value reaches $80 \%$ of the maximum. The generation of the warnings is carried by some discrete signals. Discretes can be generated in aircraft applications mainly uses the $28 \mathrm{~V} / \mathrm{OC}$ discrete generation. The $28 \mathrm{~V} / \mathrm{OC}$ discrete generation circuit is basically given as:

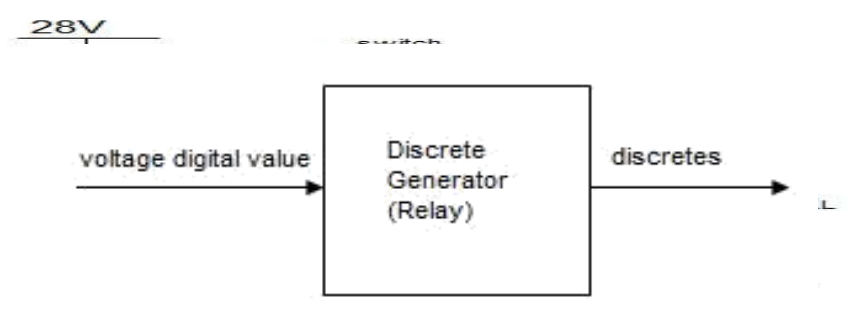

Figure 11: Schematic Diagram of 28V/OC Discrete Generation

When the switch is closed, it allows current to flow thereby generating $28 \mathrm{~V}$ at the output whereas when it is open, it gives a $0 \mathrm{~V}$. This is used in isolation with magnetic inductor or an optical diode controls the relay switch. The control and the switch are kept separated so that input logic circuits are unaffected by any current or voltage transients occurring at the output supply. For generating discretes is: 


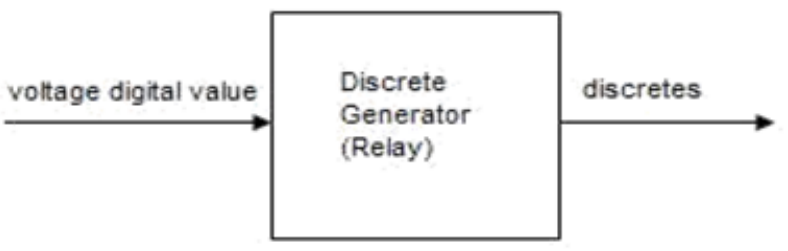

Figure 12: Block Diagram of Discrete Generation

The generation of dicretes in our application has been achieved through the use of a separate IC chip the SCD21CDY which takes in the digital voltage from the microcontroller and discrete is generated.

\section{Schematic Model}

The modelling if the integration of ARINC 429 with microcontroller MSC1212 and the Data Acquisition from the sensors has been achieved through CADSTER V1.0. The figures attached shows the modelling in details.
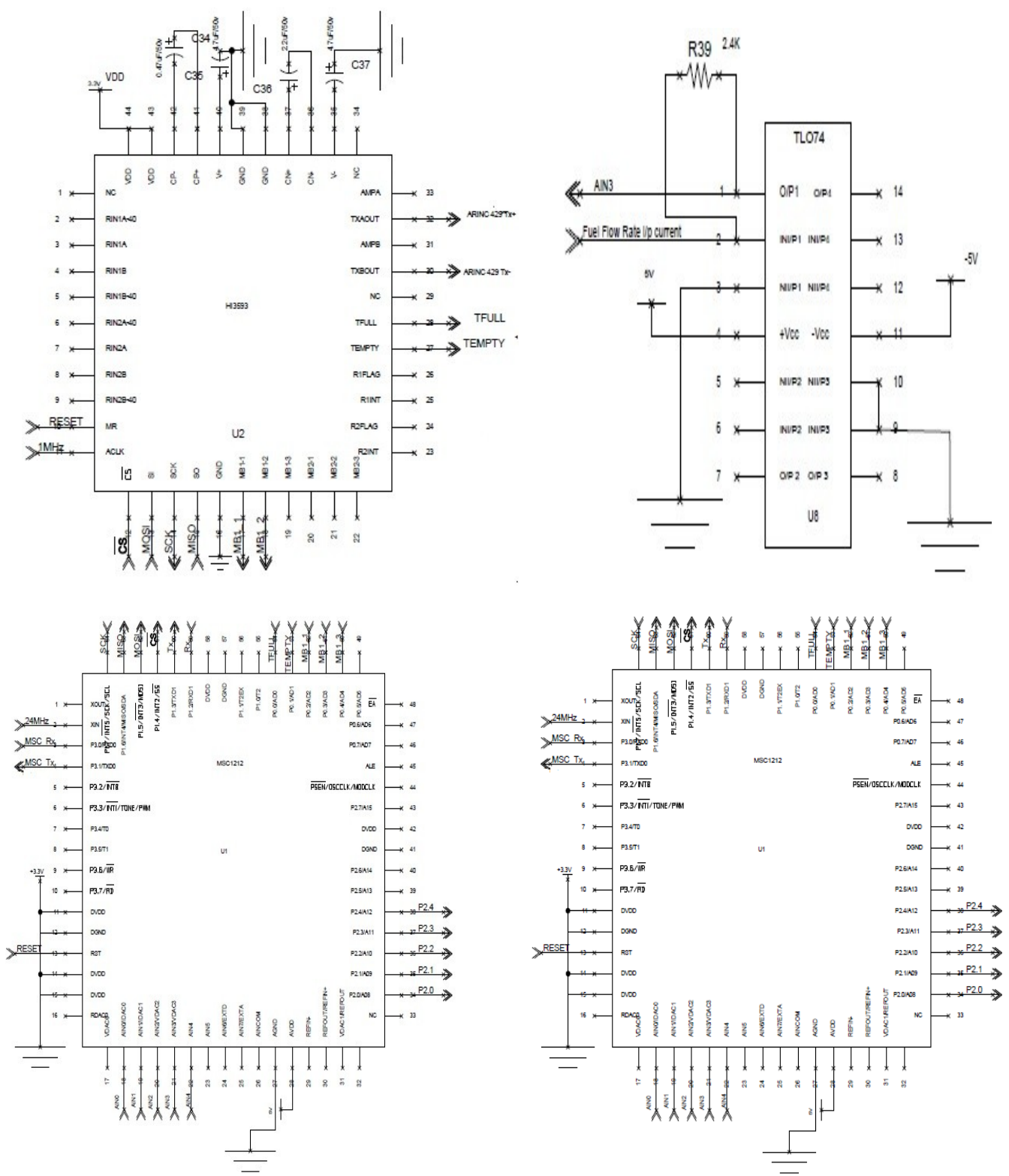


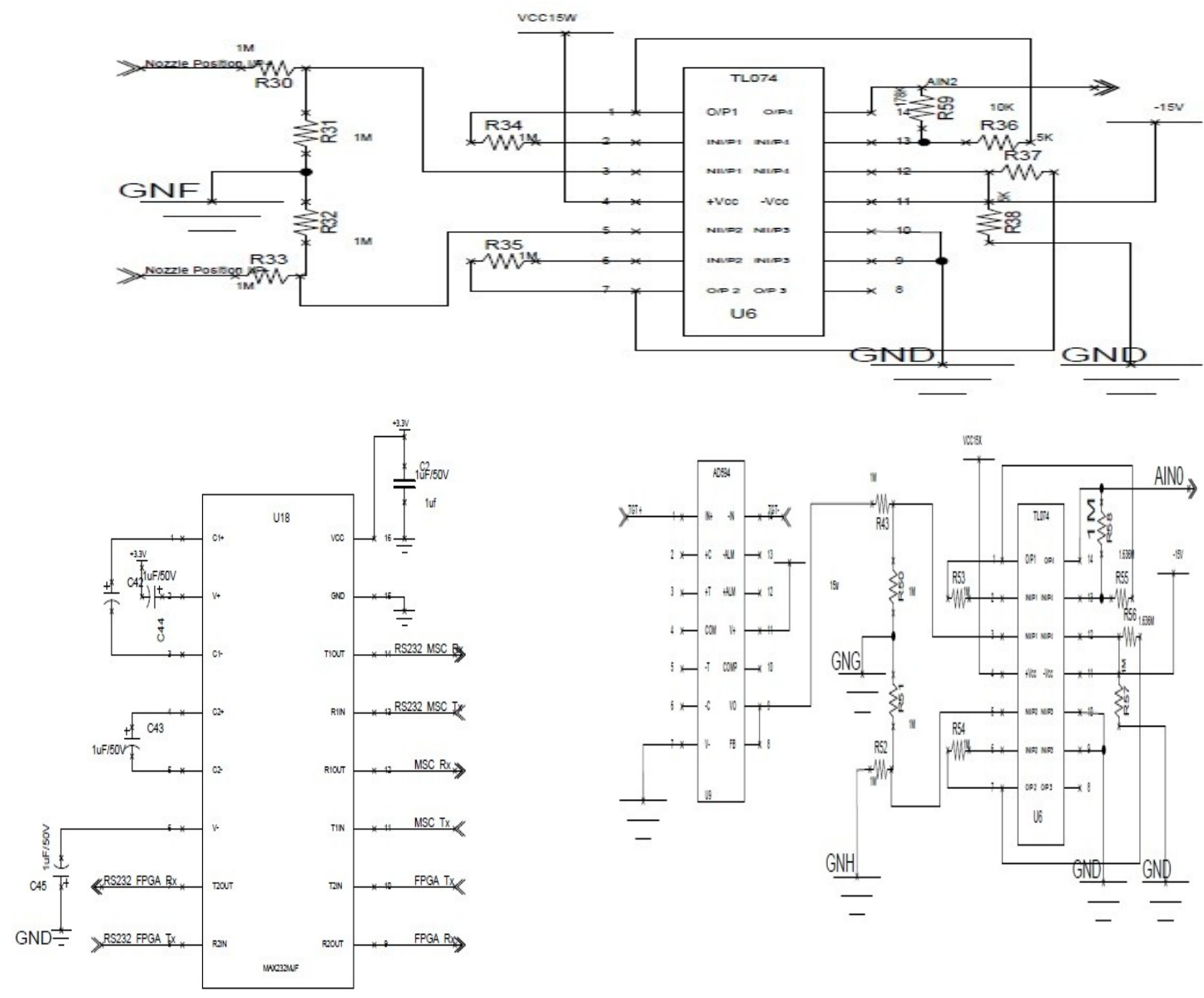

\section{Acknowledgement}

I would like to offer our heartfelt gratitude to the Mission Combat and System Research and Design Centre Department, Hindustan Aeronautics Limited, Bangalore for their guidance and constant support for the execution of the project.
[1]. Texas Instruments MSC1212(2004)
[2]. Holt Integrated Circuits Inc. HI-3593 (2013)
[3]. Analog Devices Monolithic Thermocouple Amplifiers with Cold Junction Compensation
[4]. Texas Instruments LM2907-N, LM2917-N(2013)

\section{References}

